\title{
APPLYING SEMANTIC WEB TECHNOLOGIES FOR EFFICIENT PREPARATION OF SIMULATION STUDIES IN MANUFACTURING
}

\author{
Markus Rabe \\ Technical University Dortmund \\ Leonhard-Euler-Str. 5 \\ D-44227 Dortmund, GERMANY
}

\author{
Pavel Gocev \\ Siemens AG \\ Wolfgang-Reuter-Platz 4 \\ D-47053 Duisburg, GERMANY
}

\begin{abstract}
This paper addresses methods for the preparation of simulation studies in the manufacturing domain. The approach builds on an existing Semantic Web Platform for Modeling and Simulation that supports planning and simulation projects especially during information preparation and results evaluation. A new platform module was developed in order to support simulation project members in the early phase, especially in the provision of information as well as in the rapid capacity analysis. The module integrates the constraints that have to be considered during the definition and calculation of different solution scenarios. These constraints are built as semantic rules utilizing the predicate logic and the Semantic Web technologies.
\end{abstract}

\section{INTRODUCTION}

The Procedure Model for Simulation (Figure 1) comprises two phases that are very important not just for the simulation study itself, but also can be very beneficial for a rapid capacity analysis of the system to be simulated (for a detailed discussion of the Procedure Model see Rabe et al. 2008b). The first one is the phase of System Analysis that includes the objective to determine which elements of the real system in which granularity and with which mechanism have to be modeled and simulated. The second phase is the development of the Formal Model that contains a system-independent description of the future simulation model. In the phase of turning the model description into the executable model and before the start of the implementation phase, it is very beneficial to provide the simulation expert with rough but substantial information about the performance of the system to be simulated, especially about the required capacities. The best method is to assess the planned manufacturing system in the early phase and to obtain measurable benchmarks and performance indicators of the future system like capacity demand for each resource in hours per year, suitable shift system, required number of workstations, and necessary production space for the designated production portfolio and product mix. Additionally, there is a necessity to provide different views on the real system, its structure and components, the relations between the parts' functions and the resources. These multi faceted views should support the process of communication, answering, clarification and conclusion within the project phases.

According to the Procedure Model in Figure 1, data (Raw Data and Prepared Data) are administrated separately from the knowledge (Conceptual Model and Formal Model) during simulation studies. Therefore, a significant effort has to be made in order to provide an information base for capacity estimation in the early phase of the simulation project. This paper suggests a solution for efficient and rapid preparation and support of simulation studies in the manufacturing domain. The solution is based on Semantic Web technologies as a system of ontologies and offers an explicit description of the system to be simulated beginning after the System Analysis phase integrating the data and therewith building a Manufacturing Knowledge Base (MKB). The same knowledge can be detailed within the phase of Model Formalization 
and prospectively in the phases of Implementation and Experiments and Analysis and deployed in all phases of the simulation project.

This paper first gives an overview of the related work and derives the requirements for an explicit description of manufacturing systems. Section 3 relates to the current developments of the authors regarding the Semantic Web Framework for Modeling and Simulation. The application of the Semantic Web Technologies for a rapid preparation of simulation studies and capacity estimation is explained in section 4 . The last section summaries the developments and looks beyond, towards future developments.

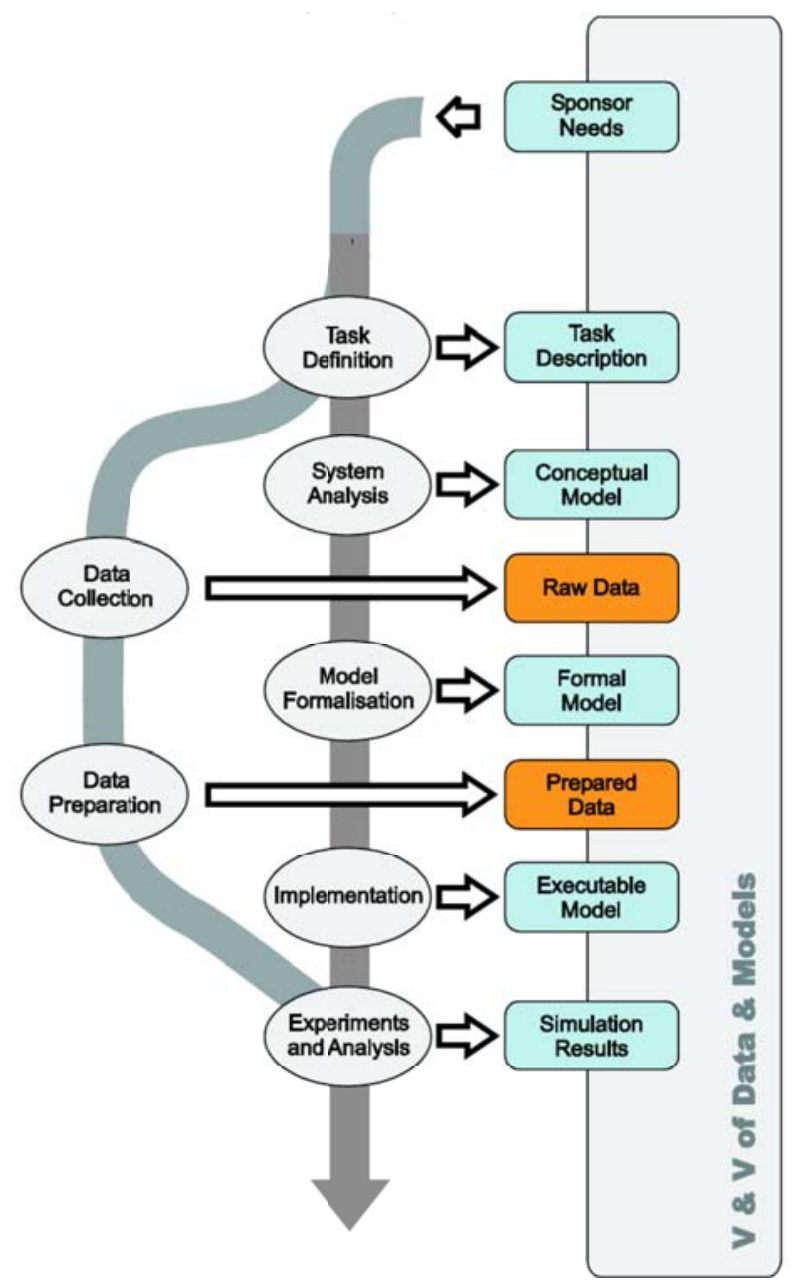

Figure 1: Procedure Model for Simulation (Rabe et al.2008a)

\section{CHALLENGES AND REQUIREMENTS ON SEMANTIC MODELS FOR SIMULATION}

The preparation of simulation studies is a process which is related to acquisition and structuring of information. A methodology for input data management in discrete event simulation projects was developed by Skoog and Johanson (2008) suggesting the steps to be performed in order to enable validated input data for discrete event simulation (DES). A very important step is the compilation of the available data where a sufficient amount of data points has to be extracted and through additional calculations converted into a suitable form for further processing. An input data management (IDM) methodology has been developed by Bengtsson et. al (2009) yielding in an architecture for generic data management (GDM) with an objective to link production data stored in different IT systems into the discrete event simulation (DES) models and convert the data according to the Core Manufacturing Simulation Data (CMSD) format de- 
veloped by SISO (2009). A chain of sub-processes within information acquisition for modeling and simulation has been developed by Kuhnt and Wenzel (2009) suggesting a very general procedure for data preparation allocating the steps of data collection, recording, structuring, statistical analysis and validation.

Especially in manufacturing workshops, structures are more simple and thus less flexible, as throughput times mainly depend on control strategies and the concrete material flow may often be neglected or strictly simplified for the simulation model. Therefore, this kind of system is a very good candidate for automatic generation of Prepared Data and of the Executable Model from the company's data. Unfortunately, the major problem is that terminology and format of the data highly differs among companies (and often, even among departments within the same company). Thus, there are good chances to solve the problem of automatic model generation for one specific case, which is repetitively applied. However, the consultant's problem having to build up a simulation model in short time to give valid advice to an enterprise is massively affected by the problem of diverse data formats and meanings.

The existing solutions consider data preparation mainly regarding the syntax of the available data and operation with databases. The next important step is to consider the meaning of the information regarding the semantics and to utilize a knowledge base. The functionalities for knowledge description and simulation of manufacturing systems that have to be provided in order to support the M\&S projects have been described by Rabe and Gocev (2008) and provided in a Semantic Web Platform for modeling, semantic enrichment of the knowledge and extraction of the necessary information for M\&S.

Ontologies have found wide applications in the last years for formalizing the semantics and thus the meaning in specifically delimited areas. However, little research has specifically dealt with semantic modeling and simulation, and the authors are not aware of any publications on the use of semantic models for simulation input data analysis in the manufacturing domain. An early and quite generic approach has been described by Miller and Baramidze (2005) who developed a Discrete-event Modeling Ontology (DeMO) that defines a model as a set of components and mechanisms, based on the Web Ontology Language (OWL, 2004). As Bell et al. (2010) clearly analyze, "a transformation directly to such a model will likely miss tacit domain concepts". Thus, this kind of ontology is suitable only as an underlying schema for an ontology that needs to be domain-specific. Consequently, the Discrete Event Simulation Component (DESC) ontology proposed by Bell et al. (2010) is specified on an example, in this case the health service simulation for the UK's National Blood Service. Those approaches care for the model semantics, but not specifically for the data. In the authors' opinion, this is at least dangerous, as (according to the system definition) the functionality cannot be fully defined without a very clear specification of input and output data. Some military approaches around the HLA (cp. Lee and Kim 2010) seem to take the data better into account, but again they focus on the components, so in this case data are meant as data around the component interfaces (in this case, exploiting HLA) and not the data exchanged with the environment through the system borders. Also, the domain concept (military) limits the applicability with respect to the manufacturing application area. None of these research approaches focuses on simulation model concepts with the "real world factory" data that form the most important basis for manufacturing simulation.

The goal of this research is to address this research field and thus to simplify and accelerate the work of the simulation consultant who has to prepare data for simulation of manufacturing workshops and to validate these data. This includes both structural information (like machines per workshop) and organizational data (like the demand for specific products, shift calendars etc.). A knowledge base is developed to align the data, interlink related information, and then to enable quick and simple calculations to validate the data.

\section{SEMANTIC WEB PLATFORM FOR MODELLING AND SIMULATION}

The backbone of the Semantic Web Framework for M\&S for the explicit description of the manufacturing domain for modeling and simulation is a Reference Manufacturing Ontology (OWL-M). The structures to model a manufacturing system are taken from the ISA-95 (ISA 2005) and OAGIS (OAGIS 2008) standards and modeled as classes, subclasses, data type properties and relations among them. The ontology can 
be populated with the instances of a particular manufacturing system, yielding the Manufacturing Knowledge Base (MKB). The knowledge base can include information from different project members contributing from several company divisions. Usually, the dispersed information within the developing projects are owned by various project members, resulting in different structures and formats. In order to enable their inclusion into the MKB, a unique syntax is needed and then a consistent structure is demanded to eliminate the inconsistencies and redundancies. Most of the applications have an export function to generate XML format. The existing XML files with different structures can be imported into one or more ontologies with their original structures. Through suitable rules these ontologies can be mapped to the OWL-M structure and populated yielding the MKB. Due to the variety of information sources, where usually the same objects are described by several people in a different way and very often not completely, the MKB comprises hidden knowledge that still has to be discovered by the user through manual search. Determined rules modeled upon the ontology can be used for inference, resulting in generated knowledge and therewith an enriched MKB. This approach can be deployed for rapid completion of the input information for the capacity analysis as well as for the performance of the simulation study.

\subsection{Reference Manufacturing Ontology (OWL-M)}

The Reference Manufacturing Ontology (OWL-M) is developed at Fraunhofer IPK upon the syntax of RDF (2004) and OWL (2004) and is an extension of the data model for simulation presented by Rabe and Gocev (2006), comprising the main classes from the ISA-95 standard:

- Process segments as a business view on the production,

- Product definition with bills of materials and production plans,

- Resources and their subclasses (personnel, equipment and material),

- Work description of production, maintenance, quality tests and inventory, related to capabilities as the highest sustainable output rate that could be achieved, schedules as the planned activities to be performed and performance as a report of the production responses.

Beside these classes, OWL-M comprises additional ones for the description of shift patterns, spatial elements for the layout, manufacturing engineering project phases (installation, qualification and ramp-up), resource status, queues, transporters and paths, performance indicators, etc. The attributes and parameters as well as the relations among the objects of the manufacturing system can be described with the properties within the ontology through the basic building block: the triple subject-predicate-object. A triple is a 3 -tuple as an ordered list of three elements. Usually the predicate can be object-typed to relate two objects, data-typed to relate object and data, and annotation-typed to annotate objects. An example triple with object-typed property is (SiP_2000 : hasPartCapacitor_7) where two objects are related with the property :haspart.

\subsection{Rule-based Mediation of Source Ontologies into the Manufacturing Knowledge Base}

The Manufacturing Knowledge Base (Figure 2) comprises content from several sources. The input information from different IT applications is imported as separate XML files. These XML files can be transformed to OWL files with a weak semantic, since they still have the same structure as in the original XML file and just use the OWL syntax. The integration of the elements within these OWL files into the structure of OWL-M can be realized through rule-based mediation. This mapping procedure yields the correspondences between the source OWL files and OWL-M. The alignment and matching of the ontologies are specifications of similarities. Those specifications are the base for rule development by the engineer. The rules govern the merging and creation of the MKB on the skeleton of OWL-M. An inference engine (software) applies the rules, reasons over the OWL-M structure, if necessary generates new classes, properties and instances and populates the existing ones in form of statements (triples). 


\section{MODULE FOR RAPID DATA PREPARATION AND CAPACITY ANALYSIS}

\subsection{Requirements for Capacity Planning in the Early Project Phases}

One of the main conclusions from manufacturing system development projects and simulation studies is that sufficient data content for capacity analysis in the early project phase is not available. The common practice is that three independent domains within the companies provide three different views and related models: process models, product definition with bill of materials, and technology description. Due to the variety of deployed IT-Applications for Enterprise Resource Planning (ERP), Manufacturing Execution Systems (MES), Product Data Management (PDM), Supply Chain Management (SCM), and Knowledge Management (KM) Systems but also numerous Content Management Systems and various Office Applications, the companies administer many islands of solutions and models that are not harmonized and contain redundant data. At the beginning of the project phases System Analysis and Data Collection, usually the following models and information are available:

- Estimated production program as a list of end products and related quantities to be delivered per year to the customers.

- Bill of Materials (BOM) for the end products or for reference products (described in 4.2).

- General Process Model of the production and the presumed technologies (described in 4.3).

- Technology descriptions and parameters that influence the process times based on the experience from previous products and existing manufacturing systems.

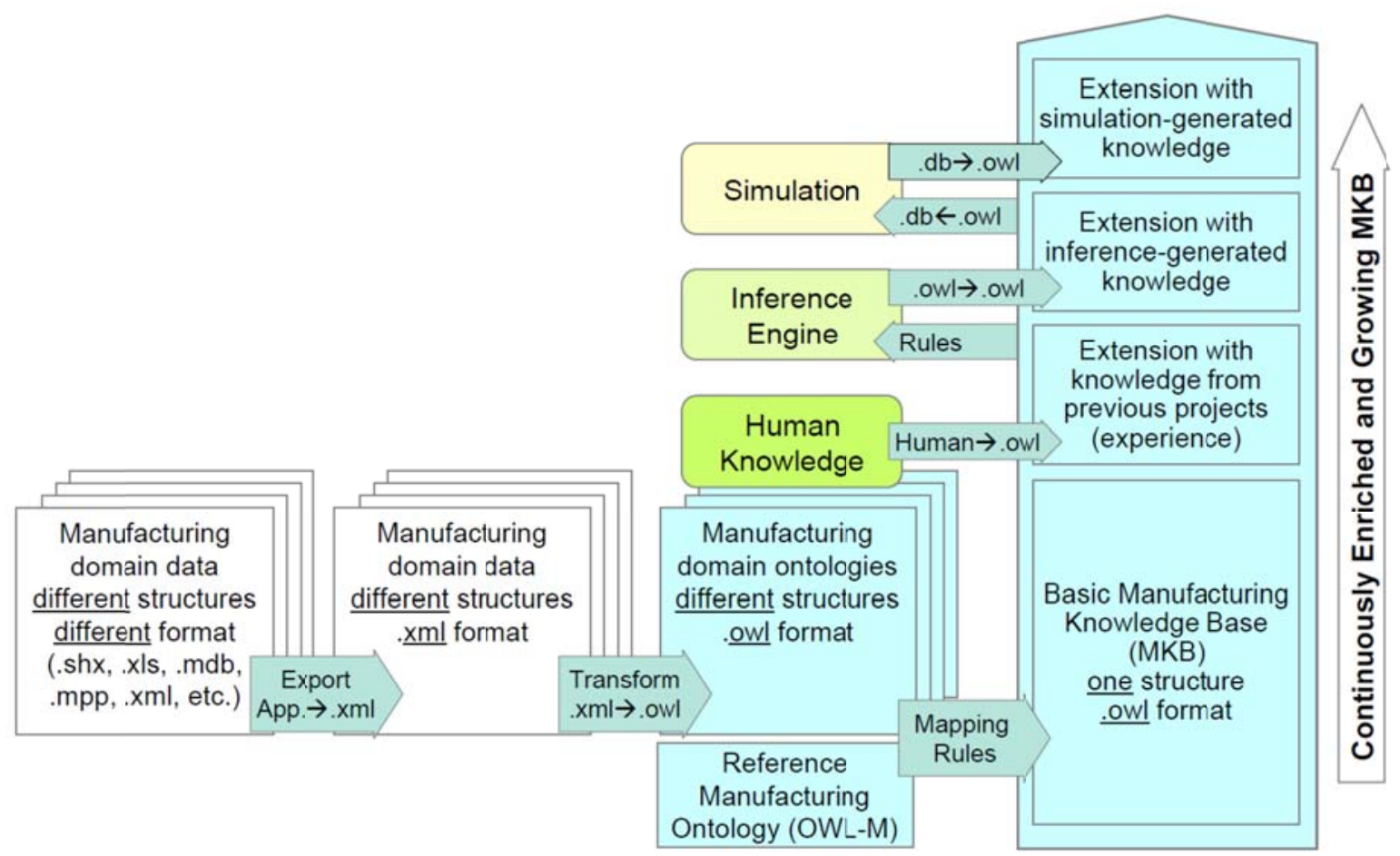

Figure 2: Semantic Web Framework for M\&S

However, this information is still not sufficient for the calculation of performance indicators to obtain a first statement about the manufacturing system capability to achieve the objectives defined with the production program. When considering the components of a simulation model given by Spur and Stöferle (1994, p. 123f) or the object model according to ISA-95, respective process plans have to be available for each product and for related components and items to be produced within the analyzed manufacturing system. The process of preparation of these information usually consumes about $40-50 \%$ of the whole 
simulation study (Rabe and Hellingrath 2001) and is characterized by intense communication within the project team, constituted especially of the product designer, the technologist, the production engineer and the simulation experts. In order to shorten activities like acquisition, modeling and completion of information within the simulation study, an automatic provision and preparation of sufficient data is needed. This challenge yields the following requirements:

- Rapid generation of quantities for all parts needed to produce the final product.

- Automated generation of process plans in the early project phase for the parts to be produced.

- Capacity analysis and manufacturing system evaluation through performance indicators.

- Formal definition of solution scenarios.

- Formal definition of the simplified decision logic for the simulation model.

The last one influences the capacity calculation just partly, but its availability is compulsory for the simulation, respectively for the phases of Implementation and Experiments and Analysis.

\subsection{Product Description by the Product Designer}

The results from the product design domain are the entirety of design information including the construction drawings and materials specification. For the phase of capacity analysis, the only necessary information is the Bill of Materials (BOM), describing the required materials and parts including the information about quantities of each part in order to build a product. The consumables and the manufacturing parameters are usually not included in the product design documentation, as these are to be defined by the technologist or production engineer during the planning phase. For the purpose of capacity estimation the BOM-structures are defined within OWL-M considering the product structure and the relations between the consisting parts (Figure 3) like :has_Child, :has_Child_Item, :has_Part and :has_Quantity.

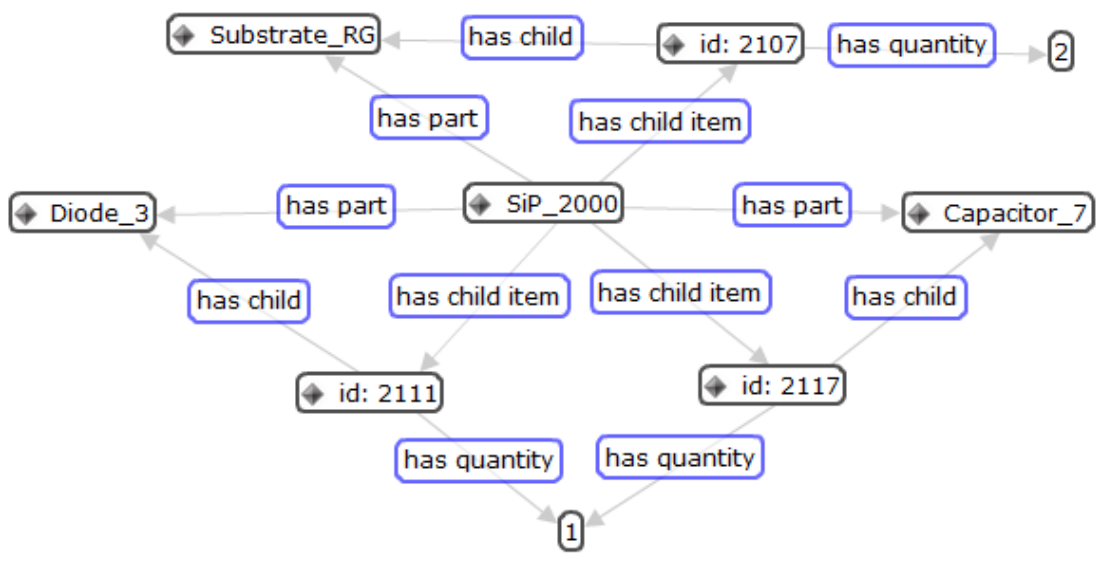

Figure 3: Bill of Material - a sample graphical view of the ontology

\subsection{General Process Model}

The skeleton for modeling of manufacturing processes is given by OWL-M where each production or logistic activity is built up as a process segment. The general process model entails a very rough description of manufacturing process segments and includes the related resources with necessary technologies for a particular product defined with properties like:

: has Successor - the following downstream process segment,

:entailsPS - a sub-process segment which the process segment is built of,

needsResource - equipment, materials, personnel, tools, fixtures, consumables needed to perform the process segment,

: deploystechnology - technology that is allocated to the process segment, 


\section{Rabe and Gocev}

The parts that are needed for the production of one component are related with the process segment with the property : needsMaterial and the output component or end product is related with :yieldsMaterial. Additional product data for the performance of the capacity analysis in the early project phase are usually not available. At most some benchmark values or limit-values are available, based on the experiences from previous or running products. But, these values might be unsuitable for the new products and the prospective system.

\subsection{Semantic Description of Technologies for Automated Generation of Process Plans}

The most influencing information for the generation of process plans is the technology description and the existing knowledge within the domains of technology and production acquired from previous projects. The praxis in manufacturing companies is that the technologists maintain a vast documentation about the technological processes, machine and equipment capabilities, manufacturing practices, quality tests, materials and about the research and development trials. Most of the information are not structured and are kept as drawings, text documents or personal spreadsheets. Moreover, there is an experience of the experts as an implicit knowledge, which is neither documented nor structured. This experience is crucial for further developments, for the generation of process plans for future products and for the project accomplishment at all. The process plan for each product type can be generated twofold. In the classical and more time-consuming process the experts participate in numerous meetings and support the project team with advice, suggestions and the provision of intangible information. This information is used by the project members and usually is built up partially, with weak structures and very often hard-coded in particular models for the individual needs of the experts. Therewith, the reusability of this knowledge cannot be assured for future developments. The better solution suggested in this paper is the automation of information administration and preparation which will enable a rapid generation of process plans and capacity estimation. The challenge and the prerequisite for this is an explicit description of

- products, processes and resources

- knowledge about technologies to be applied

The first condition is fulfilled within the Reference Manufacturing Ontology (OWL-M) mentioned above and enables a very high level of expressivity for the description of the manufacturing system objects. In order to fulfill the second prerequisite, the engineer's experience has to be turned into explicit information description. For this purpose each technology that is mentioned in the General Process Model has been described in form of statements and rules using the objects and properties within OWL-M. This explicit description enables an inference-based determination of process times and the sequence of the operations to produce a product. The rules are built of two parts in form of an IF-THEN condition claim (Table 1). The antecedent part is described as a set of triple patterns. If all patterns in this set are true, new triples according to the consequent part will be generated. Both consist of atoms built with variables, classes, properties or individuals. The notation for the description of rules can be different and arbitrary chosen between Semantic Web Rule Language (SWRL), Jena Rules or with the Protocol and RDF Query Language (SPARQL). As an example, the statement All products of type Product_P that are processed with Technology_A have to be processed in the next operation with Technology_C can be modeled by the following rule:

Table 1: IF-THEN-Condition claim in OWL-M.

\begin{tabular}{l|l} 
IF & \multicolumn{1}{c}{ Meaning } \\
\hline ?P rdf:type :Product_P & $\begin{array}{l}\text { Consider all instances (?P) from the Class Product_P } \\
\text { Consider all process segments (?PS1) that deploy Technology_A } \\
\text { ?PS1 :deploys :Technology_A } \\
\text { FP : isProducedIn ?PS1 }\end{array}$ \\
\hline THEN & $\begin{array}{l}\text { Generate a Process Segment (?PS2) } \\
\text { ?PS2 rdf:type :PrSegments }\end{array}$ Allocate the (?PS2) to the products (?P) that satisfy the IF-conditions \\
?P : isProducedIn ?PS2 & $\begin{array}{l}\text { Allocate the (?PS2) as a successor of (?PS1) that satisfy the IF-conditions } \\
\text { Allocate :Technology_C to the generated }(? P S 2)\end{array}$
\end{tabular}


The rule is used by the inference engine that infers upon the descriptions in the ontology and generates new triples. Similarly, additionally constraints can extend the rule or necessary resources can be assigned to the generated process segments in order to detail the process plans. In "real life", at least a constraint should be amended that checks if the technology $\mathrm{C}$ is already included in the plan before adding it another time.

Another very important aspect is the information about the process time for a particular product and technology or resource. For that purpose the process time has to be expressed as a function that depends on the technology, the resource and the processed part. Considering the relations between all those elements a specific process time can be calculated. As an example for the calculation of process times, Automated Optical Inspection (AOI) of panels for Surface Mounted Device (SMD) technology is discussed. The experience knowledge about this case is that the SMD is assembled on one bigger panel that can entail several printed circuit board assemblies (PCBA) dependant on the dimensions of the board and the panel. The AOI is performed with a camera flashing the surface of the assembled panel with electronic components. The process time is a function of the camera flash time, the camera width and the dimensions of the assembled panel according to the formula:

$$
\text { ProcessTime }=(\text { CameraFlashTime }+ \text { CameraMoveTime }) \times \frac{\text { Panel }_{\text {width }}}{\text { CameraSnap }_{\text {CameraSnap }}} \times \frac{\text { Panel }_{\text {length }}}{\text { Cameritis }}
$$

This knowledge can be modeled directly as a rule which can be used to calculate the process times for all possible combinations of PCBA, panel and the deployed camera type (Table 2). The specific benefit here is that the rule considers knowledge from different domains, modeled by the respective domain experts and generates new knowledge needed for the simulation study.

Table 2: Modeling a rule to calculate process times.

\begin{tabular}{l|c} 
IF & Domain as a source of the knowledge \\
\hline ?PCBA rdf:type :PCBA & Product Design \\
?PCBA : isAssembledOn ?Panel & \\
?Panel :hasWidth ?PW & Process Model \\
?Panel :hasLength ?PL & \\
\hline ?PCBA : isProcessedIn ?PS1 & Resource Description \\
?PS1 :needsResource ?M AOI & \\
\hline ?M_AOI rdf:type :AOI & \\
?MAOI :hasCamera ?Cam & Technology \\
?Cam :hasDiameter ?CamD & Rule Modeling \\
?Cam :hasFlashTime ?CamT & \\
?Cam :hasMoveTime ?CamM & \\
\hline ?function) & \\
\hline THEN (?CamT+?CamM)* (?PW/?CamD)* (?PL/?CamD) & \\
?PS1 :hasProcessTime ?PST &
\end{tabular}

With the rule above the process times for all PCBAs that are to be processed on the AOI-Machine equipped with the camera will be calculated. One comprehensive generation of process plans for all products and related components and parts is possible if all technologies are described similarly to the examples above.

\subsection{Generation of the Complete Production Portfolio}

The capacity analysis has to consider the processes to produce all components and items needed to build a final product. Through utilization of the knowledge from three domains like product design, production program planning and strategic make-or-buy decision into one particular rule, the list of all parts that have to be produced with related quantities will be generated. The rule that considers all products from the production portfolio takes the information from the BOM for each product, checks if the item is "make" or 


\section{Rabe and Gocev}

"buy" and generates a list with all components and items calculating the related quantities per year to be produced (Table 3).

Table 3: Modeling the BOM processing.

\begin{tabular}{l|c} 
IF & Domain as a source of the knowledge \\
\hline ?EndProductrdf :type :Portfolio & Production Program \\
?EndProduct : hasDemandQty ?EPQty & Bill Of Material \\
\hline ?EndProduct : haschild ?ChildID & \\
?ChildID :relatesto ?Item & Make or Buy List \\
?ChildID :hasAssemblyQty ?Qty & \\
\hline ?Item :rdf:type :MakeProducts & Rule Modeler-Ontologist \\
\hline (function) & \\
?ItemQty=?EPQty*?Qty & \\
?HEN & \\
\hline
\end{tabular}

If the relation between one particular part and corresponding process plan is established with the property : hasProcessPlan, one inverse property like : isRelatedToPart related with owl: inverseof can be used to check and prove the completeness and availability of the process plans for all parts that are needed to produce end products given by the production program.

\subsection{Capacity Estimation}

For conducting a capacity estimation, additional information is necessary about the committed capability, available capability, necessary resources, their utilization and other performance indicators relevant for the assessment of the manufacturing system. In order to derive this information, additional rules have to be deployed and therewith appropriate triples generated that are either an input for the next calculation or the result from the capacity analysis. The process of calculation and estimation of the needed capacities comprises several steps that have to be performed. As an example, the calculation of busy time for each machine or work station for the given production program is supported by rule depicted in Table 4, considering the part demand per year and the related process plan.

Table 4: Modeling the calculation of busy time per machine.

\begin{tabular}{l|c} 
IF & Domain as a source of the knowledge \\
\hline ?Item :isProcessedIn ?PS & Generated Process Plan \\
?PS : hasProcessTime ?PST & \\
?PS :needsResource ?Machine & Generated Production Portfolio \\
\hline ?Item hasDemandQty ?IQty & \\
\hline (aggregate function) & Rule Modeling \\
?Time=SUM (?IQty*?PT) & \\
THEN & \\
?Machine :hasYearDemand ?Time &
\end{tabular}

A similar logic can be used to model the relations and dependability functions for calculation and determination of other parameters and performance indicators of the manufacturing system like average throughput time, average inventories, utilization of resources and the optimal shift system.

\section{APPLICATION EXPERIENCE}

The method described in the paper has been applied in various simulation studies for industrial enterprises. A very illustrative example is the case of a turbine producer who planned a new production site that should merge the production of parts from several existing plants. In this case, also new parts had to be included in the study to take into account the upcoming products for the new plant. The data formats and terms from the previous plants, under different direction in the past, have been totally different. This was 
also caused by the fact that - according to their different specialization in their product portfolio - the single companies had developed diverging terminologies.

Applying the procedure illustrated in Figure 2, the data from the plants (mostly in XLS format) have been converted through XML to OWL. Then, the application of the Reference Manufacturing Ontology together with case-specific mapping rules (mostly identified already during the data acquisition meetings with the local engineers when clarifying the meaning of terms and data) led to the transformation into one consistent representation, independent of the data source. In some cases, missing links could be generated by the inference engine. For example, machines not specified in the process plan could be added by inference in cases where there was no doubt about the suitable machine when taking the kind of process and the part dimensions into account. This was especially helpful for the new parts, where the process plan steps had already been forecasted, but yet no assignment to specific machines had been done.

The knowledge base also supported to recognize data that had no relationships to other data or terms, and then to check the meaning and impact of these data. In consequence, some data could be identified as not relevant for the study, while others required the formulation of new rules in order to link the information with the production knowledge.

In this case the procedure was used to prepare simulation data in an efficient way, which was proven in the project post-processing phase and preparation of the lessons learned. Considering the number of production system objects like machines, product parts (BOM) and the process steps (routings) and compared to previous projects with similar complexity and number of objects, the above-described procedure enabled a reduction of the effort for data acquisition and preparation by 12 person days and therewith a shorter work package time span by about $20 \%$. This can be recognized as a significant increase of efficiency, especially as during the project (lasting for more than one year) there have been several updates of data from the plant side. After clarifying the missing rules in the first run, such updates could automatically be imported to the knowledge base.

Based on quite simple rules (e.g., on the impact of setups on the machine utilization, depending on the type of set up and lot sizes), estimates of the machine utilizations could be made before starting any simulation. Case of have over- or under-utilization could be identified and then clarified, leading to the detection of faulty input data as well as to changes of the plant design by changing the number of machines or the assignment of parts to machines. Such kind of calculation should be a standard in simulation studies, but in this case the MKB allowed for conducting this in a very efficient way, as the import of changed data from the different sources was possible with a very minor effort.

The last step (feeding back data from the simulation to the MKB) has not been utilized in this case, as the MKB for organizational reasons was not simultaneously used as the data base for production site planning, but for the simulation study only.

\section{CONCLUSION AND FUTURE DEVELOPMENTS}

A method based on Semantic Web technology for the preparation of simulation studies and early assessment of manufacturing systems has been developed. A Reference Manufacturing Ontology (OWL-M) is proposed as the backbone for an explicit and semantic description of the manufacturing system to be simulated. Besides data, OWL-M includes information, constraints and knowledge in form of rules yielding the MKB. The rules are utilized by an inference engine for a generation of new knowledge in form of triples and axioms that enrich the knowledge base. Therewith, sufficient information for an early capacity analysis can be deduced and necessary process plans based on the technology knowledge can be generated at least partially. The rules can also include functions upon which an aggregation can be performed and performance indicators of the manufacturing system can be calculated and estimated.

There are two development directions that are to be followed in order to realize a solution that can be implemented by the companies and to support their strategic decisions. The first one is to include more influencing parameters, details, information and experience that will improve and sharpen the results from the inference and calculation. The precondition for this is the availability of the information like alternative technologies, precise specification of dependencies (e.g., process time from the material structure, 
batching logic), accurate values about the availability of resources and materials, needed personnel qualifications, etc. The second development direction is towards the generalization of the logic for the simulation models. The objective is to build up the decision logic within the knowledge base as rules and to make it accessible for simulation software. With this approach the integration and sharing of simulation models between simulation software will be facilitated in a higher level of interoperability.

\section{REFERENCES}

Bell, D., S. de Cesare, M. Lycett, N. Mustafee, and S.E. Taylor. 2010. Semantic Web Service Architecture for Simulation Model Reuse. In: Organizational advancements through enterprise information systems; Emerging applications and developments edited by A. Gunasekaran and T. Shea, 129-136. New York: IGI Global

Bengtsson, N., G. Shao, B. Johansson, Y. T. Lee, S. Leong, A. Skoogh, and C. Mclean. 2009. Input data management methodology for discrete event simulation. In Proceedings of the 2009 Winter Simulation Conference, ed. M. D. Rossetti, R. R. Hill, B. Johansson, A. Dunkin and R. G. Ingalls, 13351344. Piscataway, New Jersey: Institute of Electrical and Electronics Engineers, Inc.

ISA. 2005. Enterprise Control System Integration, Part 3: Models of manufacturing operations management. Research Triangle Park (NC), Instrument, Systems and Automation Society.

Kuhnt, S., and S. Wenzel. 2009. Information acquisition for modelling and simulation of logistics networks. Journal of Simulation advance online publication, $6^{\text {th }}$ November 2009.

Lee, K., and B. Kim. 2010. A dynamic defense modeling and simulation methodology using semantic web services. Journal of Systemics, Cybernetics and Informatics 8 (2010) 2, 6771.

Miller, J.A., and G. Baramidze. 2005. Simulation and the Semantic Web. Proceedings of the 2005 Winter Simulation Conference edited by M.E. Kuhl, N.M. Steiger, F.B. Armstrong, and J.A. Joines, 2371 2377.

OAGI. 2008. Open applications group integration specification. Version 9.2. Open Applications Group. Available via http://www.oagi.org [Accessed April 4, 2010].

OWL. 2004. Web Ontology language reference. W3C Recommendation from $10^{\text {th }}$ February 2004. Available via http://www.w3.org/TR/2004/REC-owl-ref-20040210 [Accessed April 4, 2010].

Rabe, M., and P. Gocev. 2006. Simulation models for factory planning through connection of ERP and MES systems. In Simulation in Produktion und Logistik 2006, ed. S. Wenzel, 223-232. Erlangen, SCS Publishing House.

Rabe, M., and P. Gocev. 2008. Semantic Web framework for modelling and simulation of manufacturing systems. In Advances in Simulation for Production and Logistics Applications, ed. M. Rabe, 177186. Stuttgart, Fraunhofer IRB Verlag.

Rabe, M., and B. Hellingrath. 2001. Handlungsanleitung Simulation in Produktion und Logistik. Erlangen, SCS Publishing House.

Rabe, M., S. Spieckermann, and S. Wenzel. 2008a. A new procedure model for verification and validation in production and logistics simulation. In Proceedings of the 2008 Winter Simulation Conference, ed. S. J. Mason, R. R. Hill, L. Mönch, O. Rose, T. Jefferson, and J. W. Fowler, 1717-1726. Piscataway, New Jersey: Institute of Electrical and Electronics Engineers, Inc.

Rabe, M., S. Spieckermann, and S. Wenzel. 2008b. Verifikation und Validierung für die Simulation in Produktion und Logistik. Berlin, Heidelberg, Springer Verlag.

RDF. 2004. Vocabulary Description Language 1.0: RDF Schema. W3C Recommendation from $10^{\text {th }}$ February 2004. Available via http://www.w3.org/TR/2004/REC-rdf-schema-20040210 [Accessed April $4,2010]$.

SISO. 2009. Core Manufacturing Simulation Data (CMSD) information model. Draft Product Development Group, Simulation Interoperability Standards Organization. Available via http://simrest.svn.sourceforge.net/viewvc/simrest/CMSD/branches/fromUML/utils/Schema\%20for\%2 0CMSD\%20V3b [Accessed April 01, 2010] 
Skoogh, A., and B. Johansson. 2008. A Methodology for Input Data Management in Discrete Event Simulation Projects. In Proceedings of the 2008 Winter Simulation Conference, ed. S. J. Mason, R. R. Hill, L. Mönch, O. Rose, T. Jefferson, and J. W. Fowler, 1727-1735. Piscataway, New Jersey: Institute of Electrical and Electronics Engineers, Inc.

Spur, G., and T. Stöferle. 1994. Handbuch für Fertigungstechnik, Volume 6 Fabrikbetrieb. München, Wien, Carl Hanser Verlag.

\section{AUTHOR BIOGRAPHIES}

MARKUS RABE is full professor for IT in production and logistics at the Technical University Dortmund. Until 2010 he had been with Fraunhofer IPK in Berlin as head of the corporate logistics and processes department, head of the central IT department and member of the institute direction circle. His research focus is on information systems for supply chains, production planning and simulation. Markus Rabe is vice chair of the "Simulation in Production and Logistics" group of the simulation society ASIM, member of the editorial board of the Journal of Simulation, member of several conference program committees, has chaired the ASIM SPL conference in 1998, 2000, 2004, and 2008, and is local chair of the WSC'2012 in Berlin. More than 150 publications and editions report from his work. His e-mail address is markus.rabe@tu-dortmund.de

PAVEL GOCEV is since July 2012 responsible for strategic projects at Siemens AG implementing the simulation as a planning and optimization tool in the global production network. Previously he was a scientific researcher and project manager at the Fraunhofer IPK involved in various research and industrial projects. Main fields and activities are modeling, simulation and optimization of production and logistic systems. He is the architect and developer of the Semantic Web Platform for Modeling and Simulation. His email address is Pavel.Gocev@IPK.Fraunhofer.de 